\title{
Improvement of chaetominine
} production by tryptophan feeding and medium optimization in submerged fermentation of Aspergillus fumigatus CY018

\author{
Yu-peng Zhang ${ }^{1,2}$, Rui-hua Jiao ${ }^{3}$, Yan-hua Lu (1, $^{2^{*}}$ and Ling-yun Yao ${ }^{1,4^{*}}$
}

\begin{abstract}
Background: Chaetominine (CHA) is a novel alkaloid with excellent medicinal activities produced by Aspergillus fumigatus CY018. However, its further application has been severely restricted by the low production yield. In this work, the fermentation titer of CHA was investigated by medium composition optimization and amino-acid addition strategies.

Results: Under the optimized conditions of sucrose $115.03 \mathrm{~g} / \mathrm{L}$, ammonium acetate $3.98 \mathrm{~g} / \mathrm{L}$, D-tryptophan $3.84 \mathrm{~g} / \mathrm{L}$, $\mathrm{KH}_{2} \mathrm{PO}_{4} 1.5 \mathrm{~g} / \mathrm{L}, \mathrm{FeSO}_{4} \cdot 7 \mathrm{H}_{2} \mathrm{O} 0.02 \mathrm{~g} / \mathrm{L}, \mathrm{MgSO}_{4} \cdot 7 \mathrm{H}_{2} \mathrm{O} 0.7 \mathrm{~g} / \mathrm{L}$, sodium glutamate $3 \mathrm{~g} / \mathrm{L}$, sodium tartrate $1.5 \mathrm{~g} / \mathrm{L}$, and $\mathrm{CaCl}_{2}$ $0.045 \mathrm{~g} / \mathrm{L}$ ), a CHA production yield of $55.92 \mathrm{mg} / \mathrm{L}$ was obtained, which increased significantly (3.99-fold) as compared with the unoptimized basal medium. Scale-up fermentation was carried out in a 5-L bioreactor based on the shakeflask fermentation results, maximum CHA yield of $48.53 \mathrm{mg} / \mathrm{L}$ was obtained at an air flow rate of $2.0 \pm 0.1 \mathrm{WVM}$ and an agitation rate of $400 \mathrm{rpm}$.

Conclusion: These results demonstrated that medium composition optimization and amino-acid addition were useful strategies for improving CHA production via biotechnological process. The methods in this work would be useful for the biotechnological production of CHA from A. fumigatus.
\end{abstract}

Keywords: Chaetominine, Aspergillus fumigatus, Medium optimization, D-Tryptophan

\section{Background}

Many endophytic microbes have the potential to produce bioactive natural products that may directly or indirectly be used as therapeutic agents for the treatment of various diseases (Kusari et al. 2014). Chaetominine (CHA) is a bioactive alkaloidal metabolite isolated from the endophytic filamentous fungus Aspergillus fumigatus CY018 (Yao et al. 2016). The compound exhibited strong cytotoxic activity against the human leukemia K562 and

\footnotetext{
*Correspondence: luyanhua@ecust.edu.cn; lyyao@sit.edu.cn ${ }^{1}$ State Key Laboratory of Bioreactor Engineering, East China University of Science and Technology, Box 283\#, 130 Meilong Road, Shanghai 200237, People's Republic of China

${ }^{4}$ School of Perfume and Aroma Technology, Shanghai Institute of Technology, 100 Hai Quan Road, Shanghai 201418, People's Republic of China

Full list of author information is available at the end of the article
}

colon cancer SW1116 cell lines, which suggested that this bioactive metabolite might serve as a promising candidate for anti-cancer treatment (Kusari et al. 2014; Yao et al. 2016).

CHA was first reported to be a compound with new framework synthesized by endophytic Chaetomium sp. IFB-E015 in 2006 (Yao et al. 2016). Due to the unprecedented skeleton and its potential biological properties in treating cancer diseases, a number of efforts have been directed to the total synthesis of $\mathrm{CHA}$ in recent years (Snider and Wu 2007; Toumi et al. 2008; Malgesini et al. 2009; Peng et al. 2014). More recently, there are some reports indicating that endophytic Aspergillus species are also shown to produce CHA. For example, CHA is obtained from endophyte $A$. terreus isolated from stem of rice and A. fumigatus CY018 isolated from Cynodon 
dactylon (Shen et al. 2015; Liu et al. 2016). In the previous report, it was speculated that CHA might be biosynthesized from L-alanine, anthranilic acid, and D-tryptophan (Jiao et al. 2007), and the biosynthetic proposal has been practically verified by the biomimetic synthesis method (Xu et al. 2015). However, no research has focused on the bioproduction of CHA by submerged fungus cultivation, and little is known about the fermentation conditions for the improvement of its production.

The aim of this study was to develop a suitable culture medium for $\mathrm{CHA}$ production in the submerged fermentation of A. fumigatus CY018 and to evaluate its potential use for scaling-up of CHA sustainable production. The carbon and nitrogen source appropriate for fungus growth and $\mathrm{CHA}$ accumulation were first selected based on the conventional single-factor method. Secondary, the effects of amino acids, including L-alanine and D-tryptophan, which were proposed as speculated precursor units of CHA (Jiao et al. 2007), on microbial growth and CHA biosynthesis were investigated systematically. In addition, the fermentation medium was further developed and optimized by RSM for improving CHA production and scaling-up fermentation. The established medium constituents were supposed to be efficient nutritional components for the CHA production performance and would provide important support for the scaling-up production of the bioactive metabolite.

\section{Methods}

\section{Chemical and reagents}

Reagents for microorganism cultivation and product extraction, including glucose, sucrose, ammonium acetate, D-tryptophan, sodium nitrate, $\mathrm{KH}_{2} \mathrm{PO}_{4}$, $\mathrm{FeSO}_{4} \cdot 7 \mathrm{H}_{2} \mathrm{O}, \mathrm{MgSO}_{4} \cdot 7 \mathrm{H}_{2} \mathrm{O}$, sodium glutamate, sodium tartrate, methanol, and ethyl acetate were purchased from Sinopharm Chemical Reagent Company, China. Acetonitrile of HPLC grade was purchased from TEDIA Company, USA. All other chemicals used in this study were of AR grade unless indicated otherwise. Authentic $\mathrm{CHA}$ was provided by Prof. RX Tan (Nanjing University, China) and confirmed by the NMR and LC-MS techniques.

\section{Microorganisms and preparation of inoculum}

The CHA-producing strain (A. fumigates CY018) was an endophytic fungus isolated from Cynodon dactylon, which was provided by Prof. RX Tan (Liu et al. 2016). The strain was regularly maintained on potato-dextrose agar (PDA) slant and stored at $4{ }^{\circ} \mathrm{C}$. For the seed culture, the slant was inoculated in the PDA liquid medium and cultivated at $180 \mathrm{rpm}$ at $28^{\circ} \mathrm{C}$ for $48 \mathrm{~h}$.

\section{Shake-flask fermentation}

The original fermentation medium consisted of $100 \mathrm{~g} / \mathrm{L}$ of sucrose, $3.5 \mathrm{~g} / \mathrm{L}$ of sodium nitrate, $1.5 \mathrm{~g} / \mathrm{L}$ of $\mathrm{KH}_{2} \mathrm{PO}_{4}$,
$0.02 \mathrm{~g} / \mathrm{L}$ of $\mathrm{FeSO}_{4} \cdot 7 \mathrm{H}_{2} \mathrm{O}, 0.7 \mathrm{~g} / \mathrm{L}$ of $\mathrm{MgSO}_{4} \cdot 7 \mathrm{H}_{2} \mathrm{O}, 3 \mathrm{~g} / \mathrm{L}$ of sodium glutamate, and $1.5 \mathrm{~g} / \mathrm{L}$ of sodium tartrate, and the initial $\mathrm{pH}$ of the medium was adjusted to 6.0 using 1-mol/L HCl before sterilization. Shake-flask fermentation was performed by inoculating $7-\mathrm{mL}$ seed culture in a 250-mL flask containing $50 \mathrm{~mL}$ of fermentation medium. Then, the flasks were incubated at $28{ }^{\circ} \mathrm{C}$ on a rotary shaker (180 rpm) for 16 days.

\section{Medium optimization by the RSM experimental design}

Central composite design (CCD) was applied to accurately optimize the CHA production. Each independent variable was coded at three levels between -1 and +1 . The ranges of variables, including sucrose (A), ammonium acetate $(\mathrm{B})$, and $\mathrm{D}$-tryptophan $(\mathrm{C})$, are shown in Table 1 . The central concentrations were determined by preliminary experiments. Twenty experiments were augmented with six replications at the design center to evaluate the pure error and were carried in randomized order as required in many design procedures. The statistical software Design-Expert was used for the regression analysis of experimental data and to plot response surface.

The mathematical model generated during CCD implementation was validated by conducting experiment on given optimal medium setting. All data obtained in this work were the mean of triplicate experiments, and the error bars indicated the corresponding standard deviation (SD). $P$ values were used to check the significance of the differences among cultures under different conditions. A value of $P<0.05$ was considered statistically significant.

\section{Lab-scale bioreactor system experiments}

In the lab-scale bioreactor process, the experiments were performed in a 5 - $\mathrm{L}$ stirred bioreactor which equipped with two layers of six-flat-blade disk turbine impellers. 400-mL inoculum was inoculated into the bioreactor containing 2.6- $\mathrm{L}$ fermentation medium. The fungus was cultured for 16 days at $28 \pm 0.2{ }^{\circ} \mathrm{C}$ with the stirring speed of 200-500 rpm. The air flow was set at $1.5 \pm 0.1$ VVM during the whole process and $0.3 \%(\mathrm{v} / \mathrm{v})$ antifoam

\begin{tabular}{|c|c|c|c|c|}
\hline \multirow[t]{2}{*}{ Independent variables (g/L) } & \multirow[t]{2}{*}{ Symbols } & \multicolumn{3}{|c|}{ Code levels } \\
\hline & & -1 & 0 & +1 \\
\hline Sucrose & A & 80 & 100 & 120 \\
\hline Ammonium acetate & B & 3 & 3.5 & 4 \\
\hline D-Tryptophan & C & 3.3 & 4.3 & 5.3 \\
\hline
\end{tabular}


(mixture of organic polyether dispersions) was added before autoclaving.

\section{Analytical methods}

Biomass accumulation was estimated using the dry cell weight (DCW) analysis (Tey et al. 2014). A sample was filtered through a $0.45-\mu \mathrm{m}$ millipore cellulose filter that retained the hyphae. The hyphal solids were washed with sterile distilled water and dried to constant weight by air blowing thermostatic oven. Residual sugar was determined by the anthrone-sulfuric acid method (Cai et al. 2010). The broth was extracted three times with an equal volume of ethyl acetate. The upper liquor was collected and evaporated under reduced pressure. The dried crude extract was dissolved in methanol and prepared for the HPLC analysis after filtration $(0.45 \mu \mathrm{m})$.

The HPLC system of SHIMADZU LC-10Avp plus with a PDA detector (SPD-M20A) and a C18 column $(4.6 \mathrm{~mm} \times 250 \mathrm{~mm}, 5 \mu \mathrm{m}$, Agilent ZORBAX Eclipse XDB-C18) was used to analyze the concentration of CHA. The mobile phase was acetonitrile/water (35:65). The HPLC analysis was under the following condition: flow rate, $1 \mathrm{~mL} / \mathrm{min}$; column temperature, $28{ }^{\circ} \mathrm{C}$; UV wavelength, $226 \mathrm{~nm}$; and sample injection volume, $20 \mu \mathrm{L}$. The quantification of CHA in samples was based on the comparison of peak areas to the external standard.

\section{Results and discussion}

\section{The effect of carbon sources on fungus growth and CHA} production

It is well known that different types of carbon and nitrogen sources usually show great variations in microbial growth and metabolite production. The effect of carbon sources (with the same $\mathrm{C}$ molecular weight) on fungus growth and CHA production were examined by substituting glucose in the basal medium with various carbon sources. Experimental results illustrated that the greatest dry cell weight $(\mathrm{DCW})$ was obtained $(10 \mathrm{~g} / \mathrm{L})$ when maltose was used as the carbon source (Fig. 1a). According to the results shown in Fig. 1a, values of DCW are similar in the culture media containing sucrose, maltose, and glucose (control). However, a significant carbon source-related difference was observed in the production of CHA (Fig. 1a). When using soluble starch and glycerinum as carbon source, the DCW and CHA production both decreased drastically as compared with the control (Fig. 1a). The results demonstrated that carbon sources exhibited important influences on the cell growth and metabolites production, similar results were also found for other secondary metabolites production from $A$. fumigatus (Zhu et al. 2015). Among all the tested carbon sources, sucrose revealed to be more suitable for the fermentation performance and was selected for further investigation. As shown in Fig. 1b, the fermentation performance was changed under varying sucrose concentrations. The DCW and CHA production increased with the increase of carbon source concentration at the range of $60-100 \mathrm{~g} / \mathrm{L}$, but both decreased when the concentration was above $100 \mathrm{~g} / \mathrm{L}$ (Fig. 1b). According to the experimental results, the sucrose concentration of $100 \mathrm{~g} / \mathrm{L}$ was selected as the most suitable concentration for CHA production and used for the subsequent experiments.

\section{The effect of nitrogen source and concentration on fungus growth and $\mathrm{CHA}$ production}

It is well known that nitrogen is an important factor which affects secondary metabolites, especially nitrogencontaining molecules biosynthesis. Effects of different types of nitrogen source (sodium nitrate, ammonium acetate, ammonium chloride, peptone, yeast extract powder, and corn-steep liquor) on cell growth and CHA production were tested, and the corresponding results were illustrated in Fig. 2a. With respect to the cell growth and accumulation of CHA in A. fumigatus, ammonium acetate $(3.5 \mathrm{~g} / \mathrm{L})$ significantly increased the DCW and product yield by 59.86 and $25.79 \%$, respectively, in contrast with the control ( $3.5 \mathrm{~g} / \mathrm{L}$ of sodium nitrate). As described previously, ammonia is one of the predominant nitrogen sources which can be directly utilized by the microorganisms during the fermentation process (Chen et al. 2010). In addition, peptone and yeast extract powder also exhibited the improvement in fermentation performance but were less efficiency as compared with ammonium acetate (Fig. 2a). However, the CHA production decreased dramatically when corn-steep liquor or ammonium chloride was used as nitrogen source. The result suggested that ammonium acetate was a more suitable nitrogen source for the CHA production.

As shown in Fig. 2b, the DCW of fungus A. fumigatus increased with the increase concentration of ammonium acetate during the tested concentration ranges and reached maximum $(17.32 \mathrm{~g} / \mathrm{L})$ at the concentration of $4.5 \mathrm{~g} / \mathrm{L}$. The effect of ammonium acetate concentration on the production performance of $\mathrm{CHA}$ was evaluated simultaneously; the maximum fermentation yield $(31.28 \mathrm{mg} / \mathrm{L})$ was obtained at $3.5 \mathrm{~g} / \mathrm{L}$ of ammonium acetate (Fig. 2b), which was much higher than the original fermentation medium and other tested nitrogen concentration. CHA is an alkaloidal metabolite of A. fumigatus CY018 (Liu et al. 2016). Thus, there should be an intuitive connection between the nitrogen concentration and CHA accumulation. Consequently, it is not surprising that lower nitrogen concentration was negative for the CHA production. However, higher concentration of 

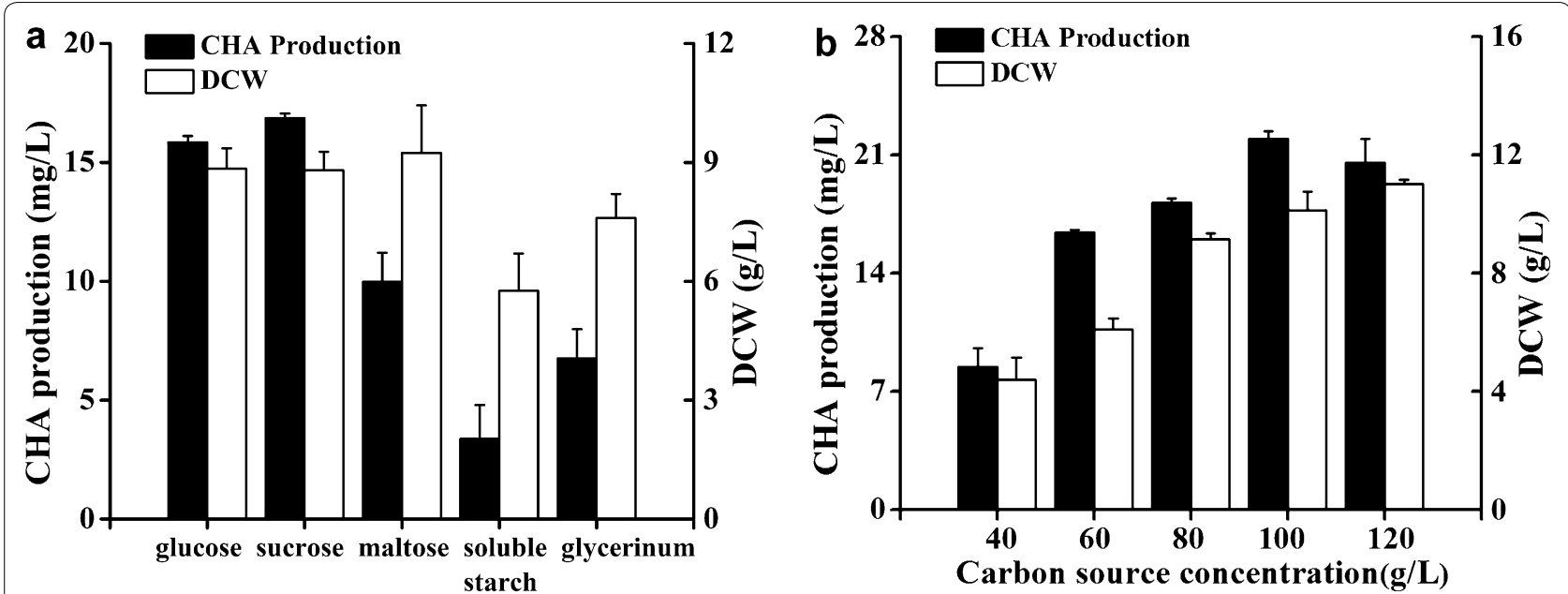

Fig. 1 Effect of various carbon sources on CHA production and DCW. a Different carbon sources of glucose, sucrose, maltose, soluble starch, and glycerinum, and $\mathbf{b}$ different sucrose concentrations
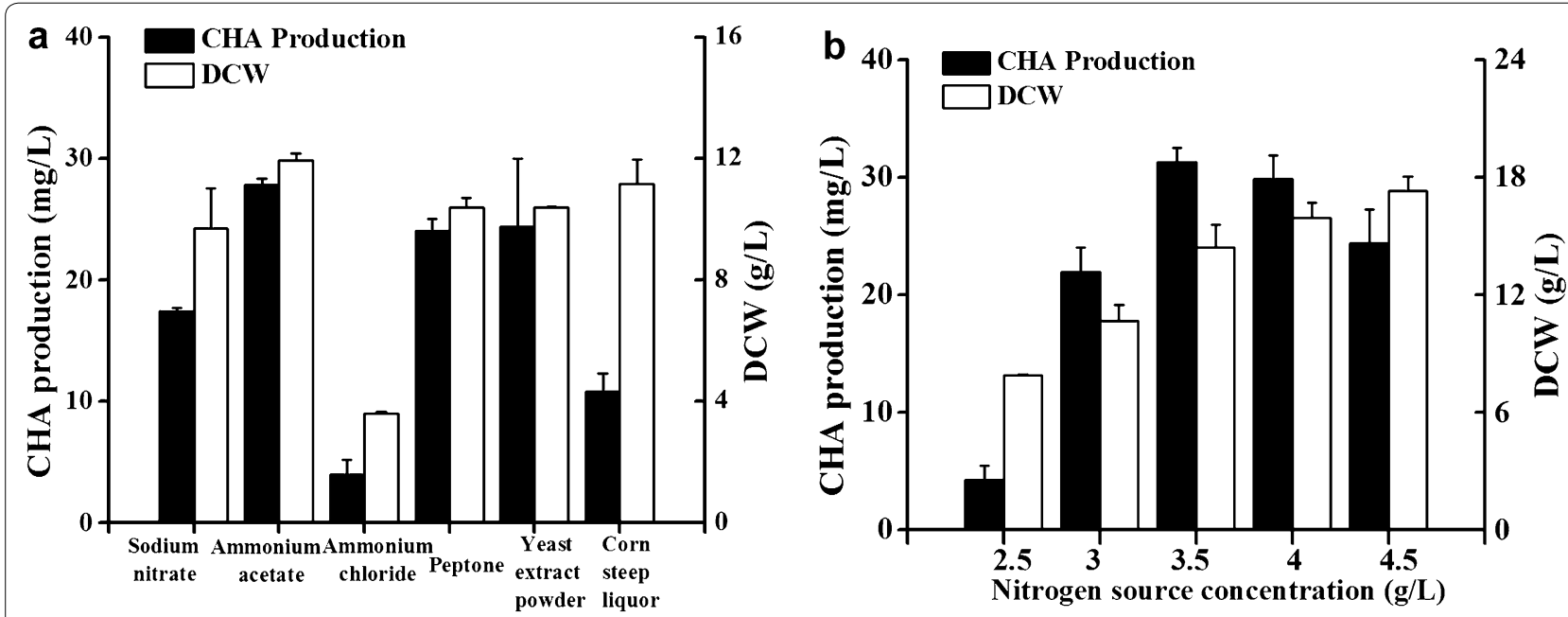

Fig. 2 Effect of various nitrogen sources on CHA production and DCW. a Different nitrogen sources of sodium nitrate, ammonium chloride, ammonium acetate, peptone, yeast extract powder, and corn-steep liquor, and $\mathbf{b}$ different ammonium acetate concentrations

ammonium acetate $(>3.5 \mathrm{~g} / \mathrm{L})$ in culture medium had a negative effect on CHA accumulation (Fig. 2b). In a previous report, inhibition of the production of secondary metabolites by high concentration of nitrogen source was also observed, which suggested the inhibition mechanism was due to the depression of enzymes in primary or secondary pathways related to the product biosynthesis (Wei et al. 2012). Among all the tested nitrogen sources and concentrations, ammonium acetate of $3.5 \mathrm{~g} / \mathrm{L}$ revealed to the most optimal condition for improving $\mathrm{CHA}$ production $(32 \mathrm{mg} / \mathrm{L})$ and was selected for further medium optimization in the subsequent experiments.
Regulation of CHA production by amino acids addition

Alkaloids are nitrogen-containing secondary metabolites which are distributed widely in plants and microorganisms, which are usually derived from amino acids, such as alanine, histidine, phenylalanine, tryptophan, and lysine (Marienhagen and Bott 2013; Xu et al. 2014). According to the speculated biosynthesis pathway (Jiao et al. 2007), D-tryptophan and L-alanine may serve as the precursors for the regulation of $\mathrm{CHA}$ production. Therefore, the two kinds of amino acids, D-tryptophan and L-alanine, were chosen to evaluate its potential effects on $\mathrm{CHA}$ accumulation. When adding L-alanine and D-tryptophan (5 and $10 \mathrm{mM}$ ) at the beginning of fermentation, the $\mathrm{CHA}$ 
accumulation declined, as shown in Fig. 3a. On the contrary, the DCW was improved slightly improved (Fig. 3a). The suppressed formation of CHA was likely caused by the inhibiting effects of L-alanine and D-tryptophan on the biosynthesis-related enzymes. A previous study has reported that amino-acid addition may inhibit the formation of related enzymes involved in the secondary metabolites biosynthesis (Yoon et al. 1995).

The addition of precursor during fermentation was supposed to be a useful experimental tool for improving product yield. Adding side-chain precursor during penicillin fermentation process to greatly increase its industrial production is the most successful example (Eriksen et al. 1994). Although the addition of speculated precursors at the beginning of fermentation showed less effective on the CHA production, however, the fermentation productivity was significantly changed by the variation of the addition time and concentration. As shown in Fig. $3 \mathrm{~b}$, the addition of $\mathrm{L}$-alanine $(10 \mathrm{mM})$ at different fermentation time caused various changes in the fermentation performance, and a maximum product yield of $34.96 \mathrm{mg} / \mathrm{L}$ was obtained at the addition time of $264 \mathrm{~h}$. By adding D-tryptophan at $192 \mathrm{~h}$ (Fig. 3c), the CHA production reached maximum value of $42.79 \mathrm{mg} / \mathrm{L}$, which was $20 \%$ higher than the optimal L-alanine addition and much higher than the control (non-addition of amino acid). The results indicated that the addition of D-tryptophan at specific fermentation time could be considered as a useful strategy for regulation of CHA production.

Thereafter, the effect of tryptophan concentration on fungus fermentation was further evaluated for improving CHA production. At fermentation time of $192 \mathrm{~h}$,
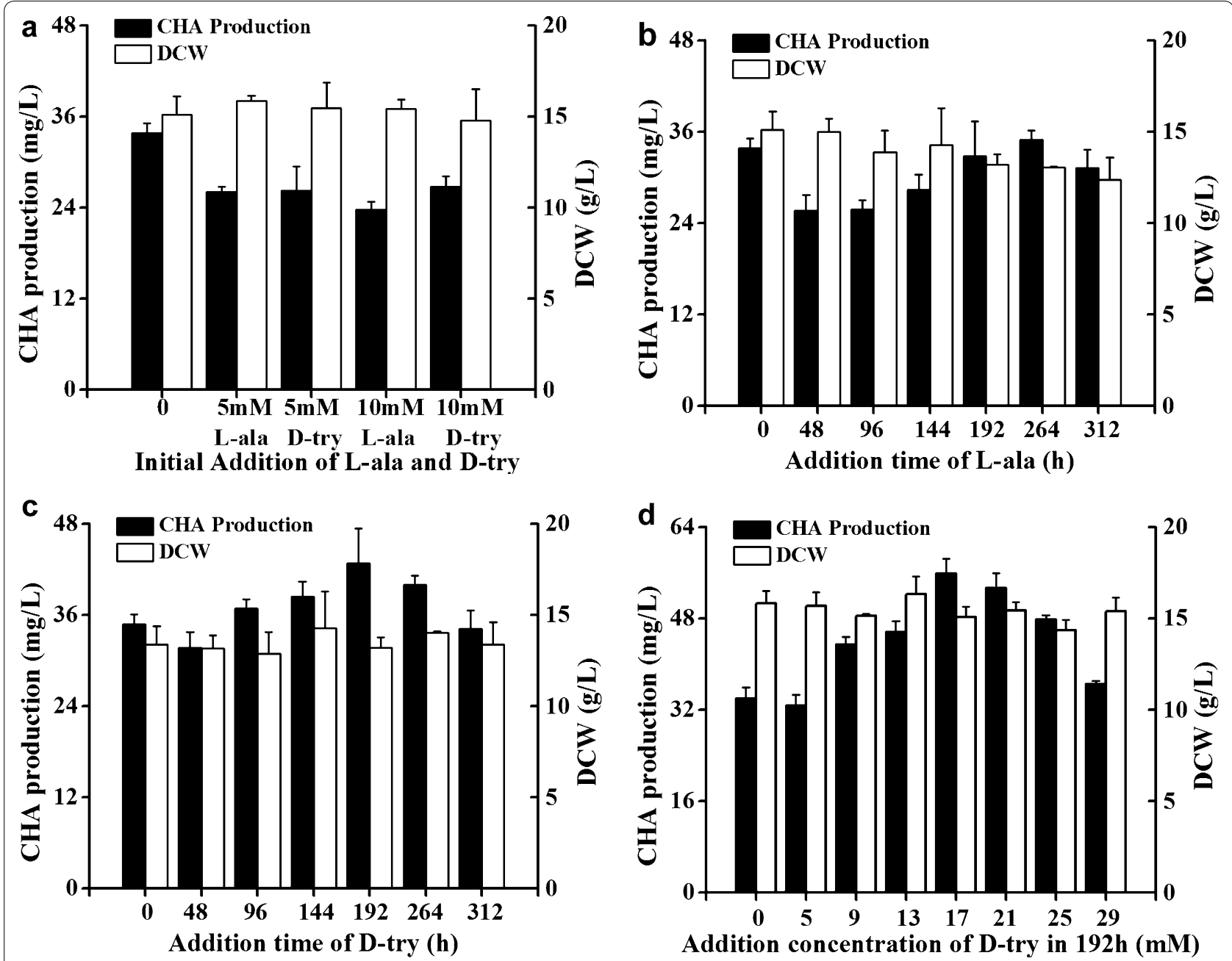

Fig. 3 Effect of D-tryptophan and L-alanine addition on CHA production and dry cell weight by Aspergillus fumigatus CY018. a The initial addition of D-tryptophan and L-alanine, $\mathbf{b}$ L-alanine addition time, with addition concentration of $10 \mathrm{mM}, \mathbf{c} \mathrm{D}$-tryptophan addition time, with the addition concentration of $10 \mathrm{mM}$, and $\mathbf{d}$ different D-tryptophan addition concentrations at $192 \mathrm{~h}$ 
adding lower concentration of tryptophan $(5 \mathrm{mM})$ slightly increased the CHA production, and higher concentrations $(9-17 \mathrm{mM})$ were more beneficial for the production performance (Fig. 3d). However, further improvement of the tryptophan concentration (21-29 $\mathrm{mM}$ ) resulted in a decreased production of CHA (Fig. 3d). Similar phenomenon was also observed in the submerged fermentation of Streptomyces sp. for dinactin production, which demonstrated that the higher doses of precursors inhibited the synthesis of dinactin (Zhou et al. 2015). In this study, the addition of $17 \mathrm{mM}$ tryptophan at $192 \mathrm{~h}$ was the most suitable feeding strategy for improving the CHA production. Under this condition, the production reached $53.93 \mathrm{mg} / \mathrm{L}$ in the shake-flask fermentation, which was 2.85 times higher than that in original medium without optimization.

\section{Medium optimization using central composite design}

After single-factor optimization experiments, the fermentation titer significantly increased as compared with the original medium. For further improving the CHA production, central composite design (CCD) was employed in this study to accurately optimize the concentration of carbon source, nitrogen source, and amino acid. The experimental results concerning CHA production using three-factor $\mathrm{CCD}$ experimental designs are shown in Table 2. The responses $\mathrm{Y}$ were fitted with the second-order polynomial equations.

$$
\begin{aligned}
Y= & 53.08-0.83 * \mathrm{~A}+2.90 * \mathrm{~B}+0.39 * \mathrm{C} \\
& +3.06 * \mathrm{AB}-1.34 * \mathrm{AC}-2.21 * \mathrm{BC} \\
& -2.98 * \mathrm{~A}^{2}-0.26 * \mathrm{~B}^{2}-3.21 * \mathrm{C}^{2} .
\end{aligned}
$$

The statistical significance of the model equation was evaluated by the $F$ test for the analysis of variance (ANOVA). The ANOVA results shown in Table 3 indicated that these quadratic models could be used to navigate the design space. The prob $>F$ values $(0.0001)$ for the CHA production are lower than 0.05 , indicating that quadratic models were significant. The relationship among the response CHA production and the two investigated factors could be represented as a response surface plot, as shown in Fig. 4. The coefficient of determination $\left(R^{2}\right)$ that was found to be closed to 1 (0.92 for $Y$ ) also advocated a high correlation between observed and predicted values. The predicted optimal condition was 115.03-g/L sucrose, 3.98-g/L ammonium acetate, 3.84-g/L D-tryptophan, and the production could be $57.57 \mathrm{mg} / \mathrm{L}$.

To verify the optimization results, experiments were performed under the predicted optimal condition. The experimental results $(55.92 \mathrm{mg} / \mathrm{L})$ closely agreed with the values obtained from RSM and, hence, validated the find-

\begin{tabular}{|c|c|c|c|c|}
\hline \multirow[t]{2}{*}{ Run } & \multicolumn{3}{|l|}{ Variables } & \multirow{2}{*}{$\begin{array}{l}\text { Response } \\
\text { CHA production (mg/L) }\end{array}$} \\
\hline & (A) Sucrose (g/L) & (B) Ammonium acetate ( $g / L)$ & (C) D-Tryptophan (g/L) & \\
\hline 1 & 100 & 3.5 & 4.283 & $53.29 \pm 1.13$ \\
\hline 2 & 100 & 3.5 & 4.283 & $53.92 \pm 0.73$ \\
\hline 3 & 100 & 2.66 & 4.283 & $46.23 \pm 2.51$ \\
\hline 4 & 100 & 3.5 & 2.603 & $43.21 \pm 0.77$ \\
\hline 5 & 66.363 & 3.5 & 4.283 & $44.92 \pm 1.16$ \\
\hline 6 & 100 & 3.5 & 4.283 & $51.79 \pm 2.05$ \\
\hline 7 & 100 & 3.5 & 4.283 & $52.82 \pm 0.12$ \\
\hline 8 & 100 & 3.5 & 4.283 & $53.62 \pm 1.71$ \\
\hline 9 & 80 & 3 & 3.284 & $43.74 \pm 0.95$ \\
\hline 10 & 120 & 4 & 3.284 & $57.82 \pm 0.21$ \\
\hline 11 & 80 & 4 & 3.284 & $43.23 \pm 2.55$ \\
\hline 12 & 133.634 & 3.5 & 4.283 & $45.38 \pm 1.42$ \\
\hline 13 & 120 & 4 & 5.282 & $47.83 \pm 0.73$ \\
\hline 14 & 80 & 4 & 5.282 & $44.93 \pm 1.34$ \\
\hline 15 & 120 & 3 & 5.282 & $44.94 \pm 0.95$ \\
\hline 16 & 100 & 3.5 & 5.963 & $45.78 \pm 2.11$ \\
\hline 17 & 120 & 3 & 3.284 & $39.78 \pm 2.76$ \\
\hline 18 & 100 & 4.341 & 4.283 & $59.42 \pm 0.22$ \\
\hline 19 & 80 & 3 & 5.282 & $47.94 \pm 0.94$ \\
\hline 20 & 100 & 3.5 & 4.283 & $52.86 \pm 1.55$ \\
\hline
\end{tabular}
ings of response surface optimization.

Table 2 Central composite design and response values 
Table 3 ANOVA analysis for responses $\mathrm{Y}[\mathrm{CHA}(\mathrm{mg} / \mathrm{L})]$

\begin{tabular}{lcccccc}
\hline Source & Sum of squares & Degree of freedom & Mean square & $\boldsymbol{F}$ value & Prob $>\boldsymbol{F}$ & $\boldsymbol{R}^{\mathbf{2}}$ \\
\hline Model & 506.99 & 9 & 56.33 & 13.64 & 0.0002 & - \\
Residual & 41.29 & 10 & 4.13 & - & -9247 \\
Lack of fit & 38.48 & 5 & 7.7 & 0.56 & - & - \\
Pure error & 2.82 & 5 & -0061 & - \\
\hline
\end{tabular}
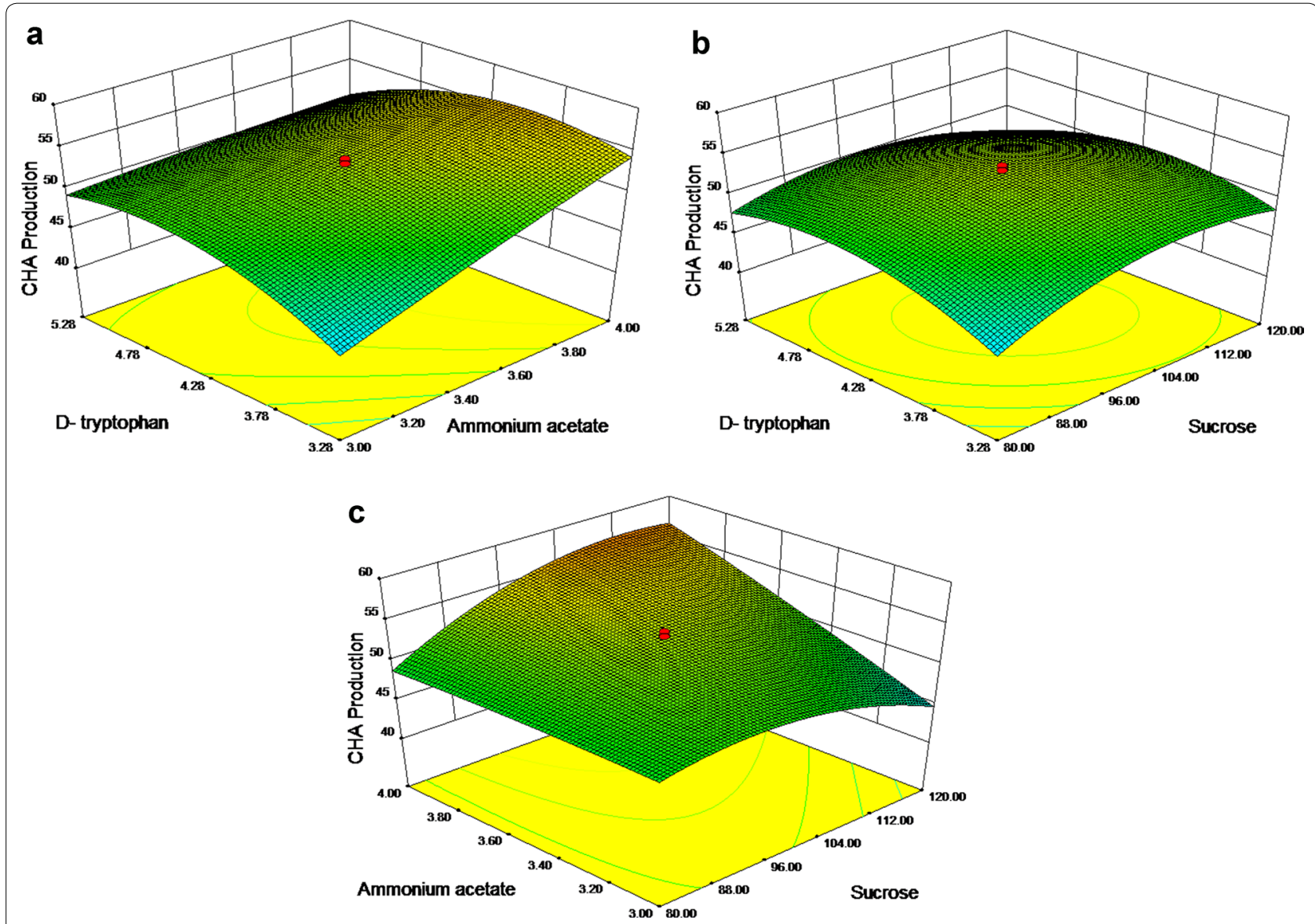

Fig. 4 Response surface plot showing the effects of two various factors on CHA production. a Combined effects on the CHA production of ammonium acetate and sucrose, $\mathbf{b}$ combined effects on the CHA production of D-tryptophan and sucrose, and $\mathbf{c}$ combined effects on the CHA production of D-tryptophan and ammonium acetate

\section{Bioreactor fermentation using the optimal medium composition and tryptophan feeding strategy}

For the scale-up production of CHA, the fermentation process was then conducted in a 5 -L stirred-tank bioreactor using the optimal medium composition and precursor feeding strategy. It has been reported that oxygen transfer is a very important factor in many fermentation processes (Hur et al. 2002). Therefore, the effect of agitation speed on CHA production in the 5-L bioreactor was investigated. The fermentation conditions were set as follows: the addition of $0.03 \%(\mathrm{v} / \mathrm{v})$ antifoam, temperature of $28 \pm 0.2{ }^{\circ} \mathrm{C}$, and air flow of $2.0 \pm 0.1 \mathrm{VVM}$. At different agitation speeds (200-500 rpm), changes of $\mathrm{CHA}$ production, cell mass, $\mathrm{pH}$, and residual glucose were observed (Fig. 5a-c). As shown in Fig. 5c, the sucrose consumption rate was relatively low at 200 and $500 \mathrm{rpm}$. Correspondingly, the cell mass (DCW) was relatively low at 200 and $500 \mathrm{rpm}$ (Fig. 5b) and CHA production was corresponding less (Fig. 5a). Comparing Fig. 5a and b, high CHA production was obtained as 

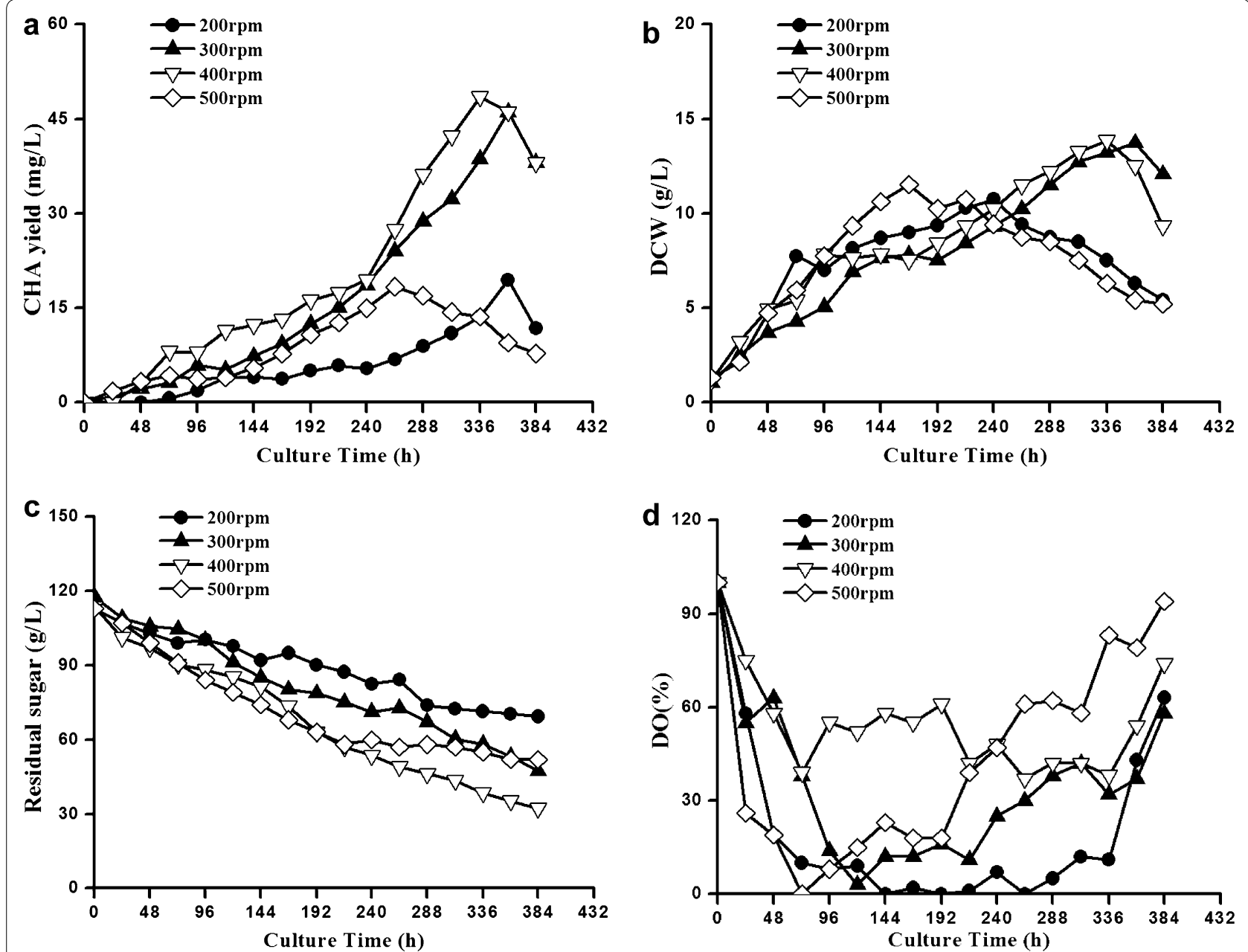

Fig. 5 5-L bioreactor fermentation of Aspergillus fumigatus CY018. Effect of agitation speeds on a CHA production, $\mathbf{b}$ cell mass, and $\mathbf{c}$ residual sugar. d Time courses at agitation speed of $400 \mathrm{rpm}$

DCW increased, which suggested that CHA production may have a positive correlation with cell mass. Lower stirring speed was harmful for oxygen and mass transfer. However, too high agitation could produce excessive shearing force and tends to be unfavorable for cell growth and CHA fermentation. In this study, the maximum cell mass concentration $(13.84 \mathrm{~g} / \mathrm{L})$ and $\mathrm{CHA}$ concentration $(48.53 \mathrm{mg} / \mathrm{L})$ were both obtained at $400 \mathrm{rpm}$ (Fig. 5a, b). Figure $5 \mathrm{~d}$ shows the time courses of CHA production at agitation speed of $400 \mathrm{rpm}$ and the production reached a maximum level of $48.53 \mathrm{mg} / \mathrm{L}$ at $336 \mathrm{~h}$. Compared with the production in original medium by bioreactor fermentation, the production yield was improved by more than $327 \%$ (data not shown). These results indicated that the optimized medium and tryptophan feeding strategy could be reproduced in scale-up fermentations for improving the CHA production.

\section{Conclusion}

In this work, the fermentation medium optimization for improving CHA production in submerged fermentation of A. fumigatus was investigated. Sucrose and ammonium acetate were selected as preferable carbon and nitrogen sources for the CHA production. In addition, adding tryptophan, a speculated precursor $\mathrm{CHA}$, at fermentation time of $192 \mathrm{~h}$ showed great improvement in the production performance. After carbon source, nitrogen source, and tryptophan addition optimization by RSM, the CHA production could reach $55.92 \mathrm{mg} / \mathrm{L}$ in the shake-flask fermentation, which was 3.99 -fold to the 
initial production. Based on the results in flask shaker, the fermentation process was successfully scaled-up in a lab-scale bioreactor, in which the CHA production could reach $48.53 \mathrm{mg} / \mathrm{L}$. The information obtained demonstrated that the optimized fermentation process is an easy and effective method for improving and scale-up production of CHA.

\section{Abbreviations}

CHA: chaetominine; DCW: dry cell weight; RSM: response surface methodology; CCD: central composite design; ANOVA: analysis of variance.

\section{Authors' contributions}

YPZ was in-charge of the experiments and paper writing. $\mathrm{RHJ}$ offered experimental strain. LYY participated in the experiments and paper writing. YHL directed the study as the tutor. All authors read and approved the final manuscript.

\section{Author details}

${ }^{1}$ State Key Laboratory of Bioreactor Engineering, East China University of Science and Technology, Box 283\#, 130 Meilong Road, Shanghai 200237, People's Republic of China. ${ }^{2}$ Shanghai Collaborative Innovation Center for Biomanufacturing Technology, 130 Meilong Road, Shanghai 200237, People's Republic of China. ${ }^{3}$ State Key Laboratory of Pharmaceutical Biotechnology, Institute of Functional Biomolecules, Nanjing University, Nanjing 210093, People's Republic of China. ${ }^{4}$ School of Perfume and Aroma Technology, Shanghai Institute of Technology, 100 Hai Quan Road, Shanghai 201418, People's Republic of China.

\section{Acknowledgements}

This work was supported by the National High Technology Research and Development Program of China (2013AA092901) as well as the National Special Fund for State Key Laboratory of Bioreactor Engineering (2060204).

\section{Competing interests}

The authors declare that they have no competing interests.

Received: 25 May 2016 Accepted: 27 August 2016

Published online: 15 September 2016

\section{References}

Cai MH, Zhou XS, Zhou JS, Niu CP, Kang L, Sun XQ, Zhang YX (2010) Efficient strategy for enhancing aspergiolide A production by citrate feedings and its effects on sexual development and growth of marine-derived fungus Aspergillus glaucus. Bioresour Technol 101:6059-6068

Chen GQ, Fan KW, Lu FP, Li Q, Aki T, Chen F, Jiang Y (2010) Optimization of nitrogen source for enhanced production of squalene from thraustochytrid Aurantiochytrium sp. New Biotechnol 27:382-389

Eriksen SH, Jensen B, Schneider I, Kaasgaard S, Olsen J (1994) Utilization of side-chain precursor for penicillin biosynthesis in a high-producing strain of Penicillium chrysogenum. Appl Microbiol Biotechnol 40:883-887
Hur BK, Cho DW, Kim HJ, Park Cl, Suh HJ (2002) Effect of culture conditions on growth and production of docosahexaenoic acid (DHA) using Thraustochytrium aureum ATCC 34304. Biotechnol Bioprocess Eng 7:10-15

Jiao RH, Xu S, Liu JY, Ge HM, Hui D, Chen X, Zhu HL, Tan RX (2007) Chaetominine, a cytotoxic alkaloid produced by endophytic Chaetomium sp. IFB-E015. Org Lett 8:5709-5712

Kusari S, Satpal S, Jayabaskaran C (2014) Biotechnological potential of plantassociated endophytic fungi: hope versus hype. Trends Biotechnol 32:297-303

Liu CQ, Jiao RH, Yao LY, Zhang YP, Lu YH, Tan RX (2016) Adsorption characteristics and preparative separation of chaetominine from Aspergillus fumigatus mycelia by macroporous resin. J Chromatogr B 1015:135-141

Malgesini B, Forte B, Borghi D, Quartieri F, Gennari C, Papeo G (2009) A straightforward total synthesis of (-)-chaetominine. Chemistry 15:7922-7929

Marienhagen J, Bott M (2013) Metabolic engineering of microorganisms for the synthesis of plant natural products. J Biotechnol 163:166-178

Peng QL, Luo SP, Xia XE, Liu LX, Huang PQ (2014) The four-step total synthesis of (-)-chaetominine. Chem Commun 50:1986-1988

Shen L, Zhu L, Luo Q, Li XW, Xi JQ, Kong GM, Song YC (2015) Fumigaclavine I, a new alkaloid isolated from endophyte Aspergillus terreus. Chin J Nat Med 13:937-941

Snider BB, Wu XX (2007) Synthesis of (-)-chaetominine. Org Lett 9:4913-4915

Tey BT, Yong KH, Ong HP, Ling TC, Ong ST, Tan YP (2014) Optimal conditions for hepatitis B core antigen production in shaked flask fermentation. Biotechnol Bioprocess Eng 9:374-378

Toumi M, Couty F, Marrot J, Evano G (2008) Total synthesis of chaetominine. Org Lett 10:5027-5030

Wei ZH, Bai LQ, Deng ZX, Zhong J (2012) Impact of nitrogen concentration on validamycin a production and related gene transcription in fermentation of Streptomyces hygroscopicus 5008. Bioprocess Biosyst Eng 35:1201-1208

Xu W, Gavia DJ, Tang Y (2014) Biosynthesis of fungal indole alkaloids. Nat Prod Rep 31:1474-1487

Xu D, Liang KJ, Tong XG, Ding M, Li DS, Xia CF (2015) Biomimetic synthesis of (-)-chaetominine epimers via copper-catalyzed radical cyclization. Tetrahedron 71:3699-3704

Yao JY, Jiao RH, Liu CQ, Zhang YP, Yu WG, Lu YH, Tan RX (2016) Assessment of the cytotoxic and apoptotic effects of chaetominine in a human leukemia cell line. Biomol Ther 24:147-155

Yoon JS, Kim JY, Rhee YH (1995) Effects of amino acid addition on molar fraction of 3-hydroxyvalerate in copolyester of 3-hydroxybutyrate and 3-hydroxyvalerate synthesized by Alcaligenes sp. SH-69.J Jerment Bioeng 80:350-354

Zhou J, He H, Wang XL, Lu J, Zhou XS, Cai MH, Zhang YX (2015) Optimization of nutrients for dinactin production by a marine Streptomyces sp. from the high latitude arctic. Biotechnol Bioprocess Eng 20:725-732

Zhu YX, Hu WW, Yao LY, Yu WG, Jiao RH, Lu YH, Tan RX (2015) Improvement of Fumigaclavine $\mathrm{C}$ production in a two-stage culture of Aspergillus fumigatus with molasses as a cost-effective ingredient. Biotechnol Bioprocess Eng 20:1106-1113

\section{Submit your manuscript to a SpringerOpen ${ }^{\circ}$ journal and benefit from:}

- Convenient online submission

- Rigorous peer review

- Immediate publication on acceptance

- Open access: articles freely available online

- High visibility within the field

- Retaining the copyright to your article

Submit your next manuscript at $\boldsymbol{\nabla}$ springeropen.com 